\title{
ADICIÓN DE POLIELECTROLITOS SOBRE LA FASE ACUOSA DE UN SUELO SULFATADO ÁCIDO INTERIOR DE CORDOBA, COLOMBIA
}

\section{EFFECT OF THE ADDITION OF POLIELECTROLYTE ON AQUEOUS PHASE OF ACID SULFATE SOILS}

\author{
Jaime L. Mercado ${ }^{1}$ Enrique M. Combatt ${ }^{2}$ y Manuel S. Palencia ${ }^{3 *}$
}

Recibido para publicación: Enero 30 de 2015 - Aceptado para publicación: Mayo 7 de 2015

\begin{abstract}
RESUMEN
El uso de polímeros, en particular polielectrolitos (PELs), en suelos se ha enfocado principalmente en el incremento de la capacidad de almacenamiento y uso eficiente del agua, aumento de la velocidad de infiltración, reducción de la frecuencia de irrigación, control de la erosión, disminución de las perdidas por escurrimiento de aguas y aumento de su utilidad para la siembra de cultivos. El objetivo de este trabajo fue determinar el efecto de la adición de polielectrolitos sobre la composición química de la fase acuosa del suelo de Suelos Sulfatados Ácidos (SSA). Para eso, SSA diferentes fueron recolectadas e incubadas con soluciones acuosas de diferentes polímeros: poli(ácido acrílico) (PAA), poli(vinilpirrolidona) (PVP) y poli(estiren sulfonato de sodio) (PSSNa). El efecto de los PELs fue dependiente de la naturaleza química de los grupos funcionales sobre la cadena del polímero. En el caso del PSSNa y el PAA, el efecto de la concentración del polímero se caracterizó por la inmovilización de los iones; mientras que para la PVP se observó un efecto solubilizante. Los resultados indican que la interacción entre las especies químicas de la fase acuosa y los grupos funcionales del polímero, es un factor importante que debería considerarse. Además, el efecto de los polímeros fue influenciado por el $\mathrm{pH}$ y los contenidos de materia orgánica.
\end{abstract}

Palabras Clave: Solución del suelo, polímero soluble en agua, suelos sulfatados ácidos, polielectrolito.

\begin{abstract}
The use of polymers in soils have been mainly focused in the increasing of water storage capacity and efficient water using, increasing of infiltration rate, reduction of irrigation frequency, erosion control, decreasing of lost by runoff and increase its usefulness for planting crops. The aim of this work was to determine the effect

\footnotetext{
${ }^{1}$ Químico, MsC. Universidad de Córdoba. Carrera 8a No 16- 23 Barrio Lacharme. Montería, Colombia

${ }^{2}$ I.A. Ph.D. Departamento de Ingeniería Agrícola y Desarrollo Rural, Facultad de Ciencias Agrícolas,Universidad de Córdoba. Carrera 12 No 54-58. Montería, Colombia.

${ }^{3 *}$ Químico, Ph.D. Departamento de Química, Facultad de Ciencias Naturales y Exactas, Universidad de Valle, Calle 13 N 100-00, Edif. 320 Ofic. 2063 Sede Melendez. Cali, Colombia. (57-2) 3212180, Fax3212128, manuel.palencia@correounivalle.edu.co
} 
of polyelectrolyte (PEL) addition on chemical composition of soil aqueous phase of acid sulfate soils (ASS). For that, different soil samples were collected and incubated with aqueous polymer solutions: poly(acrilic acid) (PAA), poly(vinyl pyrrolidone) (PVP) and poly(sodium styrenesulfonate) (PSSNa). Effect of PEL was seen to be strongly dependent on chemical nature of functional groups on polymer chains. In the case of PSSNa and PAA, effect of polymer concentration was characterized by the immobilization of ions; whereas for PVP a solubilization effect was observed. Results indicate that interaction between chemical species from aqueous phase and polymer functional groups is an important factor that should be considered. In addition, effect of polymers is influenced by $\mathrm{pH}$ and organic matter contents.

Key words: Soil solution, water-soluble polymer, acid sulfate soils

\section{INTRODUCCIÓN}

Los polímeros usados para el acondicionamiento del suelo pueden ser: Hidrogeles y polímeros solubles en agua. De manera general, los hidrogeles incrementan la cantidad disponible de humedad en la zona radicular permitiendo intervalos más grandes de irrigación y uso eficiente del agua (Callebaut et al. 1979; Li et al. 2014). Por otro lado, los polímeros solubles en agua pueden ser utilizados para incrementar la fertilidad de los suelos al actuar como transportadores de fertilizantes $u$ otras especies activas. En zonas tropicales, como resultado de la intensa lluvia, los suelos sufren una disminución en la estabilidad de sus agregados y un incremento en la densidad aparente, en consecuencia, el almacenamiento de agua se reduce y el drenaje se incrementa. En estas condiciones de alta precipitación, polímeros acondicionadores del suelo se han utilizado para el control de la erosión y para evitar las pérdidas de la capa de suelo fértil, ocasionada por la Iluvia (Callebaut et al. 1979; Wallace y Wallace 1986; Mohammed et al. 2013; Li et al. 2014). Otros efectos benéficos que han sido reportados son: aumento en la velocidad de germinación y emergencia de plantas de maíz, lechuga, tomate (Guiwei et al. 2008; Mohammed et al. 2013).
La velocidad de germinación y emergencia de un número de plantas se incrementa significativamente por la presencia de este tipo de acondicionadores. En general, el efecto benéfico de los polímeros sobre propiedades agrícolas de los suelos se ha explicado mediante aspectos como: mejor aireación del suelo y por lo tanto incremento de la actividad microbiana, retraso en la disolución de los fertilizantes, incremento en la capacidad de absorción y favorecimiento del consumo de algunos elementos nutrientes por las plantas (Guiwei et al. 2008; Dorraji et al. 2010; Mohammed et al. 2013).

Los polímeros también se han utilizado en aplicaciones encaminadas a la recuperación de suelos contaminados por metales pesados. En principio, la acción de los polímeros con este fin no radica en la disminución del contenido total de metales tóxicos, sino más bien su aplicación va encaminada en producir un cambio de su disponibilidad y por lo tanto una disminución de su efecto negativo. Esta aplicación se soporta en que los factores que rigen el flujo de metales desde la fase sólida hacia la solución del suelo gobiernan su biodisponibilidad (Guiwei et al. 2008). 
Los factores que determinan la efectividad de los polímeros como acondicionadores del suelo están íntimamente relacionados a las propiedades físicas y químicas de éstos, por ejemplo, polímeros que puedan resultar efectivos en suelos ácidos, no necesariamente lo serán en suelos salinos. Por lo anterior, es necesario que estos sean evaluados dependiendo de las características edafológicas de cada zona (Wallace y Wallace 1986; Guiwei et al. 2008, Dorraji et al. 2010; Mohammed et al. 2013). Por otro lado, los Suelos Sulfatados Ácidos (SSA) son suelos que poseen extrema acidez, un porcentaje de saturación bases intercambiables menor al $50 \%$ y aluminio intercambiable mayor de 50 $\mathrm{mmol}^{(+)} / \mathrm{kg}$ de suelo; esta acidez es desarrollada naturalmente como resultado de cambio en los niveles hidrológicos y por la oxidación de material pirítico (Powell y Martens 2005); Combatt et al. 2008; Burton et al. 2008; Li et al. 2014).

En el mundo existen entre 12 - 20 millones de hectáreas de SSA. Cerca de 6 millones se encuentran en climas tropicales húmedos del sureste asiático. Otras regiones donde esos suelos están presentes son Australia, Suecia, Finlandia, Japón, Corea, Brasil, Venezuela y Colombia (en los departamentos de Boyacá, Córdoba, y toda la zona que conforma el delta del Orinoco); (Powell y Martens 2005); Combatt et al. 2008; Burton et al. 2008; Li et al. 2014). En el departamento de Córdoba existen aproximadamente 20.000 hectáreas con SSA, no aptas para las actividades agrícolas y pecuarias, por existir condiciones químicas muy adversas como son: alta acidez, alto contenido de aluminio, formación de sales tóxicas y principalmente rangos excesivos de azufre que evitan el normal desarrollo de los cultivos (Combatt et al. 2004; Pardo et al. 2009)

En la actualidad, no existen estudios del uso de acondicionadores poliméricos sobre este tipo de suelos, por ello, investigaciones encaminadas a determinar las posibles aplicaciones de este tipo de macromoléculas en SSA pueden ayudar a vislumbrar alternativas de remediación que permitan su uso agrícola y pecuario.

El objetivo del presente trabajo fue evaluar el uso de poli(ácido acrílico) (PAA), poli(estiren sulfonato de sodio) (PSSNa) y poli(vinilpirrolidona) (PVP) sobre la fase acuosa de SSA característicos del departamento de Córdoba, Colombia, contribuyendo de esta forma, con el entendimiento del efecto que tiene la incorporación de PELs sobre las propiedades químicas de este tipo de suelos.

\section{MATERIALES Y MÉTODOS}

PAA (250 kDa, Aldrich), PSSNa (200 kDa, Aldrich) y PVP (200 kDa, Aldrich) se utilizaron como polímeros acondicionadores solubles en agua. Detalles de las metodologías de extracción y cuantificación utilizadas en cada una de las técnicas se encuentran disponibles en el manual de análisis de suelos del Instituto Geográfico Agustín Codazzi (IGAC 2006).

Resulta apropiado resaltar algunas propiedades generales de los polímeros seleccionados. Estos son polímeros lineales, homopolímeros de alto peso molecular (> $100 \mathrm{kDa}$ ), con capacidad de retener iones en solución acuosa. Dos de ellos son aniónicos (PAA y PSSNa) con 
capacidad de interactuar preferiblemente con cationes, de los cuales el PAA es un PEL débil (análogo a los ácidos húmicos) mientras que el PSSNa es un PEL fuerte (no forma equilibrio en solución acuosa). El PVP es neutro, posee una alta solubilidad en agua y es sensible a la acidez del medio que puede promover la protonación del nitrógeno del anillo pirrolidona. Las estructuras de los polímeros utilizados se muestran en la figura 1 .

\section{Recolección y pretratamiento de muestras}

La recolección de las muestras se realizó en un punto identificado previamente como suelo sulfatado ácido en el transecto San Carlos - Carrillo - Cotorra en el departamento de Córdoba (Combatt et al. 2008). Se recolectaron $2 \mathrm{~kg}$ de muestras del primer horizonte. Posteriormente, las muestras fueron secadas al aire, molidas (VEB Thurm DDR-GDR) y tamizadas por una malla de $2 \mathrm{~mm}$ (Soilstest ASTM E-11) para lograr su homogenización.

\section{Acondicionamiento de muestras}

El acondicionamiento de las muestras de SSA se realizó mediante la adición de soluciones de PAA, PSSNa y PVP a diferentes concentraciones.
Las concentraciones de polímero fueron 2, 4, 6, 8 y $10 \mathrm{mgL}^{-1}$ por cada $100 \mathrm{~g}$ de suelo. El volumen de solución de polímero se calculó de forma tal que se mantuviera constante la cantidad de polímero en las muestras, para lo cual, se determinó el volumen de saturación de cada una de ellas.

\section{Caracterización de la fase sólida}

Las muestras de SSA iníciales y acondicionadas con los polímeros fueron caracterizadas mediante la determinación de los siguientes parámetros: $\mathrm{pH}$ (método potenciométrico), acidez intercambiable (método de Yuang), fósforo extraíble (método de Bray II), porcentaje de carbono orgánico (método de WalkeyBlack), azufre extraíble (método del fosfato monocálcico monobásico), micronutrientes $\left(\mathrm{Fe}^{3+}, \mathrm{Cu}^{2+}, \mathrm{Mn}^{2+}\right.$ y $\mathrm{Zn}^{2+}$ por el método del doble ácido y cuantificándolos por Espectroscopia de Absorción Atómica, EAA, usando un equipo Perkin-Elmer 3110), bases intercambiables $\left(\mathrm{Mg}^{2+}, \mathrm{Ca} 2+\mathrm{EAA}\right.$ y $\mathrm{K}^{+}, \mathrm{Na}^{+}$por EEA los cuales fueron extraídos por el método del acetato de amonio 1,0 $\mathrm{mol}^{\mathrm{L}-1} \mathrm{a} \mathrm{pH} 7.0$ ) (IGAC 2006).<smiles>CC(C)(C)CC(c1ccc(S(=O)(=O)O[AlH2])cc1)C(C)(C)C</smiles>

PSSNa

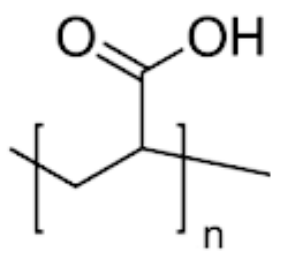

PAA

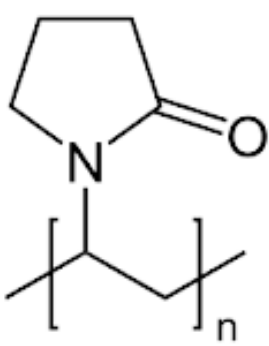

PVP

Figura 1. Estructura química de los PEL seleccionados. 


\section{Caracterización de la fase acuosa}

La fase soluble de las muestras de SSA iníciales y acondicionadas con PELs se caracterizó mediante la determinación de los siguientes parámetros: $\mathrm{pH}$ (método potenciométrico), conductividad eléctrica (CE, por el método conductidimetrico), análisis de aniones solubles $\left(\mathrm{SO}_{4}{ }^{2-}\right.$ mediante espectroscopia de absorción molecular, $\mathrm{Cl}$. mediante titulación argentométrica, y $\mathrm{CO}_{3}^{2-}$ y $\mathrm{HCO}_{3}^{-}$mediante volumetría de neutralización) y análisis de cationes solubles $\left(\mathrm{Fe}^{3+}, \mathrm{Mn}^{2+}, \mathrm{Mg}^{2+}, \mathrm{Ca}^{2+}, \mathrm{K}^{+}\right.$ y $\mathrm{Na}^{+}$por EAA en los modos de absorción y de emisión en el caso de $\mathrm{Na}^{+}$y $\mathrm{K}^{+}$, (PerkinElmer 3110); además se determinó la acidez intercambiable $\left(\mathrm{Al}^{3+}+\mathrm{H}^{+}\right)$por volumetría de neutralización (IGAC 2006). Las muestras se analizaron por triplicado en un diseño de bloques casualizado, donde cada serie o bloque de muestras a analizar estaba compuesto por cada repetición de cada uno de los tratamientos. Los datos fueron analizados mediante la prueba de media de tuky $(p<0.05)$

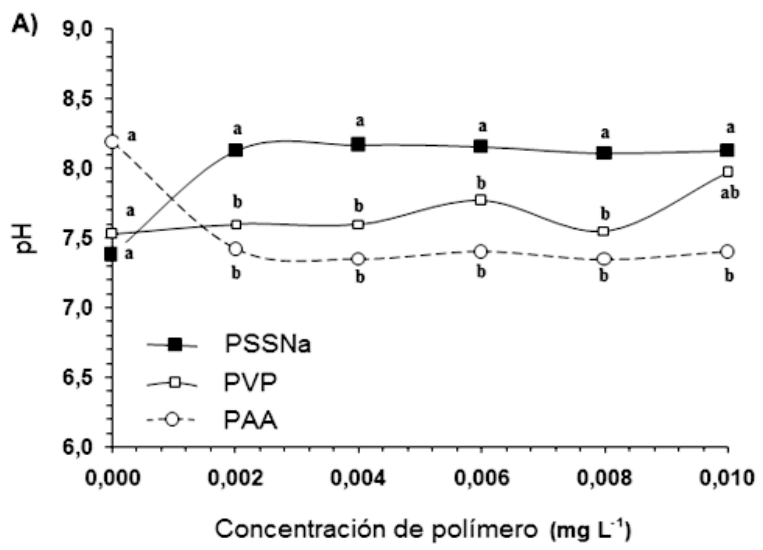

\section{RESULTADOS Y DISCUSIÓN}

A partir de los análisis de caracterización se estableció que la muestra del SSA presentó un $\mathrm{pH}$ de 4,79; un contenido de materia orgánica del 1,8\%, una CE de 0,511 dS/m, un contenido de azufre de 299,5 $\mathrm{mg} \mathrm{kg}^{-1}$ y valores de $\mathrm{Ca}^{2+}$, $\mathrm{Mg}^{2+}, \mathrm{K}^{+}, \mathrm{Na}^{+}$y $\mathrm{Al}^{3+}$ de 3,$2 ; 10,8 ; 0,82 ; 0,19$ y $4,2 \mathrm{cmol}^{(+)} \mathrm{kg}^{-1}$, respectivamente. Debido a que los análisis de la solución del suelo en presencia de las muestras modificadas y sin modificar fueron realizados bajo las mismas condiciones de saturación de agua, es de esperarse que los cambios en la composición de la fase acuosa sean principalmente resultado de la incorporación de los polímeros. El efecto de la adición de los polímeros sobre el pH del extracto de saturación y la conductividad eléctrica se muestra en la figura 2.

Se observó un efecto del proceso de la adición de agua al suelo posterior al pretratamiento; esto se concluye a partir de la comparación de los valores iníciales del $\mathrm{pH}$ y la conductividad de los experimentos de control; respectivamente los valores fueron $7,68 \pm 0,40$ y $0,45 \pm 0,11 \mathrm{dS} / \mathrm{m}$.

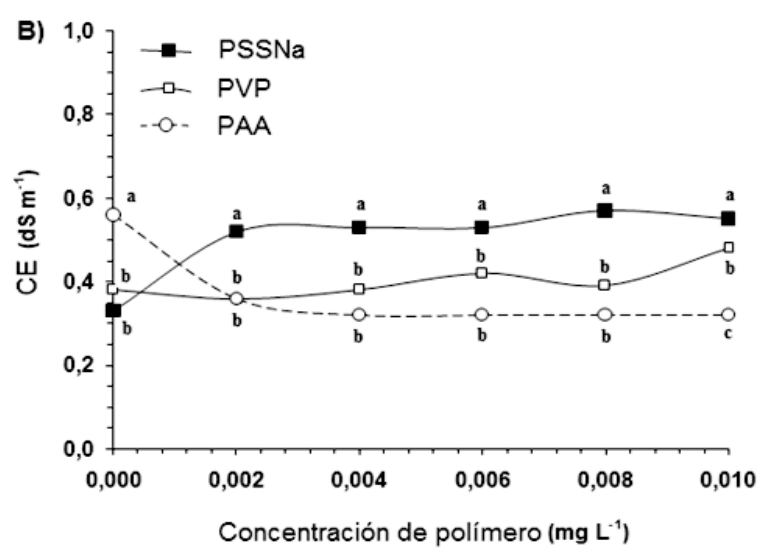

* Letras similares en orden vertical no varían estadísticamente según la prueba de media de Turkey $(p \leq 0,05)$

Figura 2. Efecto de la incorporación de los polímeros sobre el pH (A) y (B) sobre la conductividad eléctrica (CE) de la solución del suelo. 
En relación al $\mathrm{pH}$, en el caso del PAA se observó una disminución de la acidez de la fase acuosa; mientras que en el caso del PSSNa y PVP se observaron aumentos, siendo para la adición de PVP solo un incremento muy leve. El comportamiento de la CE fue similar al del $\mathrm{pH}$.

En los casos del PAA y el PSSNa, aunque ambos polímeros son ácidos su comportamiento fue completamente diferente, la diferencia puede explicarse por la naturaleza de los grupos sobre la cadena principal del polímero, en el caso del PAA los grupos ácidos carboxílicos tienden a formar complejos con $\mathrm{Ca}^{2+}, \mathrm{Mg}^{2+}$ y $\mathrm{Al}^{3+}$ originándo la disminución del $\mathrm{pH}$ y CE. Por otro lado, el PSSNa al poseer grupos ácidos fuertes no forma complejos, por lo tanto los iones presentes en la solución del suelo no forman enlaces localizados y por ende,
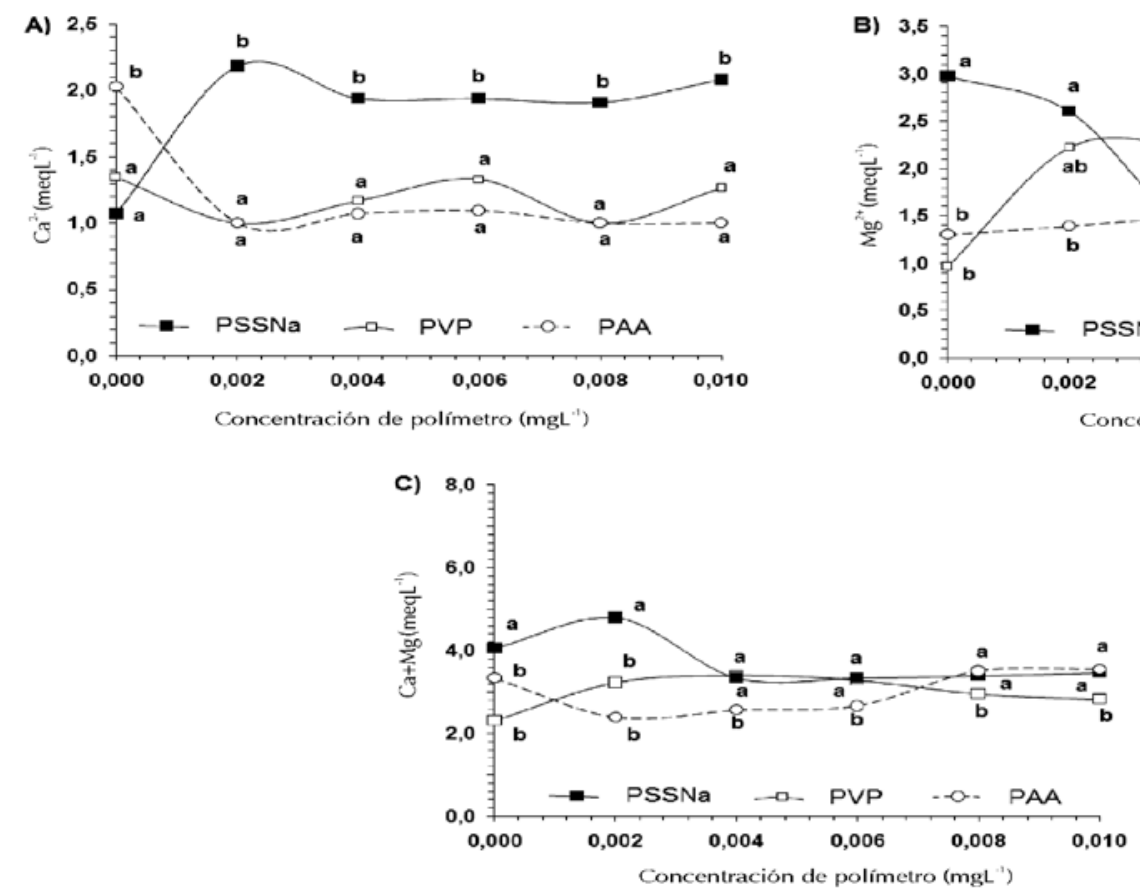

* Letras similares en orden vertical no varían estadísticamente según la prueba de media de Turkey $(p \leq 0,05)$

Figura 3. Efecto de la incorporación de los polímeros sobre los contenidos de $\mathrm{Ca}^{2+}(\mathrm{A}), \mathrm{Mg}^{2+}(\mathrm{B})$ y los contenidos totales de $\left(\mathrm{Ca}^{2+}+\mathrm{Mg}^{2+}\right)$ de la solución del suelo.(C) 
con PAA y PVP los cuales son polímeros capaces de formar enlaces de coordinación con el $\mathrm{Ca}^{2+}$. Sin embargo, para las muestras tratadas con PSSNa los contenidos aumentaron. Para los contenidos de $\mathrm{Mg}^{2+}$, los valores fluctúan evidenciando una interacción del $\mathrm{Mg}^{2+}$ con todos los polímeros.

Debido a que la química de los cationes $\mathrm{Ca}^{2+}$ y $\mathrm{Mg}^{2+}$ es similar, además de poseer igual carga neta, estos son iones de metales alcalinotérreos, y dado que su interacción con los polímeros trabajados es muy semejante, resulta conveniente analizar el comportamiento total del $\mathrm{Ca}^{2+} \mathrm{Mg}^{2+}$. Al analizar los contenidos totales de cationes divalentes $\mathrm{Ca}^{2+}$ y $\mathrm{Mg}^{2+}$ se encontró un ligero aumento de sus contenidos con la PVP y una disminución con el PSSNa.
En el caso del PAA se observó una fluctuación con una disminución de la concentración a bajos contenidos de polímero y ningún efecto apreciable a altos contenidos de polímero.

Las variaciones de los contenidos de $\mathrm{Na}^{+}$y $\mathrm{K}^{+}$ en función de la concentración de polímero se muestran en la figura 4. Los contenidos de $\mathrm{K}^{+}$para el PSSNa disminuyeron levemente, mientras que para PVP la disminución de la concentración de potasio fue más marcada, y con la adición de PAA, los contenidos de $\mathrm{K}^{+}$ fueron incrementados.

Los contenidos de sodio disminuyeron cuando PAA fue adicionado e incrementaron levemente en las muestras con PVP y PSSNa. Cuando se analizó el comportamiento del total de iones
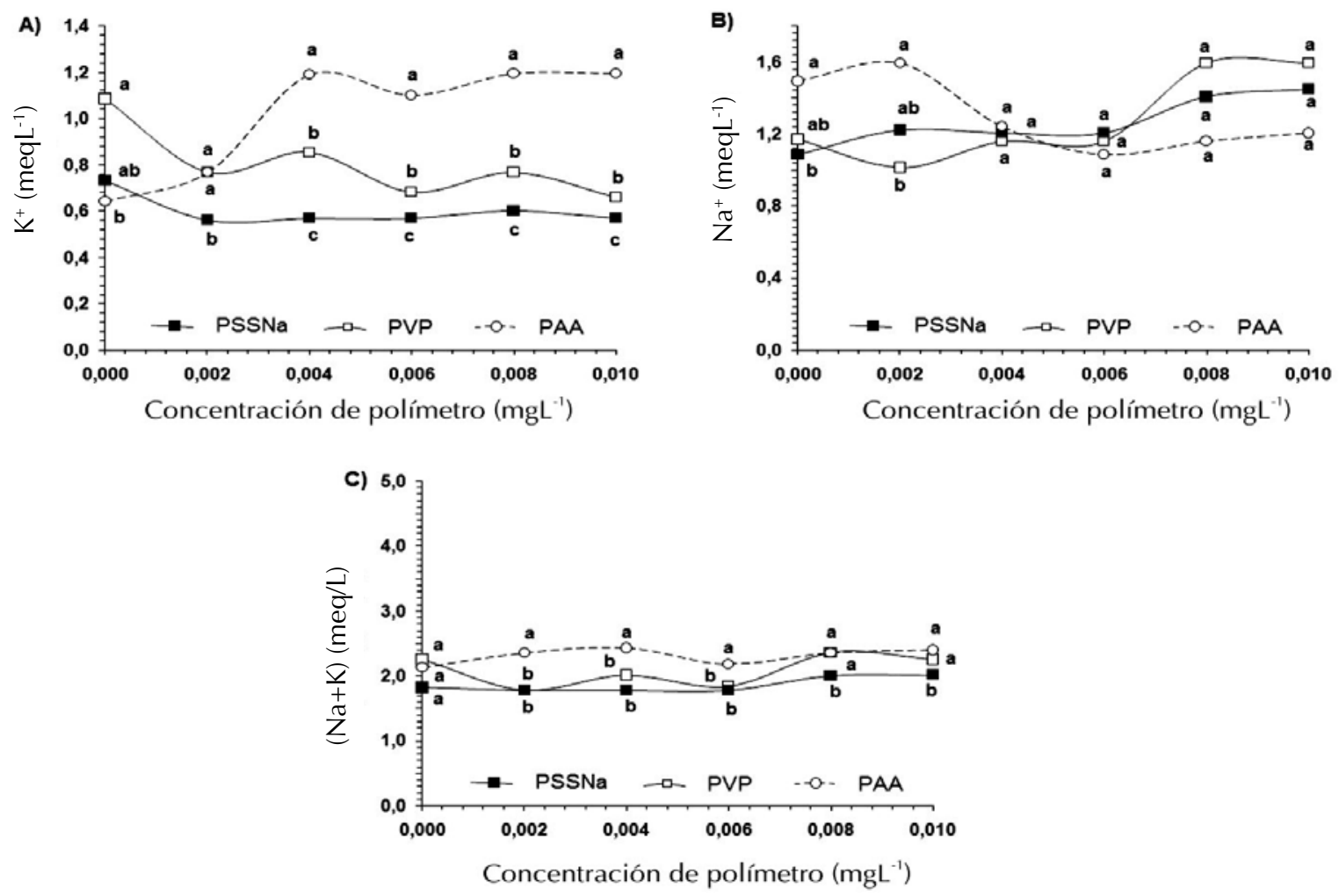

* Letras similares en orden vertical no varían estadísticamente según la prueba de media de Turkey $(p \leq 0.05)$

Figura 4. Efecto de la incorporación de los polímeros sobre los contenidos de $\mathrm{K}^{+}(\mathrm{A}), \mathrm{Na}^{+}(\mathrm{B})$ y los contenidos totales de $\left(\mathrm{Na}^{+}+\mathrm{K}^{+}\right)$de la solución del suelo $(\mathrm{C})$. 
monovalentes $\left(\mathrm{Na}^{+}+\mathrm{K}^{+}\right)$se concluyó que el efecto global es insignificante en todos los casos.

Los efectos de la incorporación de polielectrolitos a los SSA puede ser entendida mediante las diferentes interacciones de los grupos funcionales de los polímeros con especies de carga opuesta presentes. Diversos autores han sugerido que, el principal efecto de los polielectrolitos como el PAA, PSSNa y el PVP sobre las propiedades químicas del suelo están directamente relacionadas con su capacidad de actuar como intercambiadores iónicos (Mukhopadhyay et al. 1994; Wallace y Terry 1998; Bai et al. 2015) y su capacidad de adsorción sobre la superficie de las arcillas (Wallace y Terry 1998).

La incorporación de PSSNa al SSA evidencia la liberación de $\mathrm{Ca}^{2+}$ y adsorción de $\mathrm{Mg}^{2+}$ a medida que los contenidos de polímero aumentan. Así mismo, de manera menos marcada, los contenidos de $\mathrm{K}^{+}$disminuyen y los de $\mathrm{Na}^{+}$se incrementan. Por consiguiente, los resultados sugieren que la interacción entre los grupos sulfónicos $\left(-\mathrm{SO}_{3}^{-}\right)$y cationes divalentes es más favorable que la interacción con iones monovalentes. Desde el punto de vista electrostático, considerando los radios iónicos hidratados de los iones (Tabla 1), se obtiene que la densidad de carga de los iones sigue el siguiente orden: $\mathrm{Ca}^{2+}>\mathrm{K}^{+}>$ $\mathrm{Mg}^{2+}>\mathrm{Na}^{+}$, concluyéndo que la naturaleza electrostática de la interacción polímero-ión es preponderante solo con el sodio.

En el caso de las especies divalentes y el potasio, teniendo en cuenta que los grupos sulfónicos son ácidos fuertes y que por consiguiente la magnitud de su carga solo se ve afectada por la fuerza iónica, los resultados sugieren que la competencia con otras especies aniónicas presentes mayoritariamente (aniones sulfatos) en este tipo de suelos, promueve la formación de especies de menor solubilidad con especies divalentes como el $\mathrm{Ca}^{2+}$ y el $\mathrm{Mg}^{2+}$ (Rivas et al. 2011; Szilagyi et al. 2014).

El efecto del PAA, PSSNa y PVP puede estar relacionado con el peso molecular del polímero y su conformación (lineal o entrecruzada), densidad de carga, y naturaleza de los grupos funcionales sobre la cadena principal (Mukhopadhyay et al. 1994; Bai et al. 2015). La alta solubilidad de los polímeros PAA y PSSNa y su alta capacidad de absorción de agua e iones sobre su superficie sugiere que es posible esperar un efecto marcado bajo las condiciones experimentales (bajas concentraciones y tiempos de incubación relativamente pequeños) (Rivas et al. 2011; Szilagyi et al. 2014; Bai et al. 2015)

Tabla 1. Tabla 1. Radios iónicos hidratados (Rhid) y densidad de carga efectiva (dQef) de los iones $\mathrm{Ca}^{2+}, \mathrm{Mg}^{2+}$, $\mathrm{Na}^{+}$y K $\mathrm{K}^{+}$(Nightingale 1959).

\begin{tabular}{ccccc}
\hline Catión & $\mathbf{C a}^{2+}$ & $\mathbf{M g}^{2+}$ & $\mathbf{N a}^{+}$ & $\mathbf{K}^{+}$ \\
$\mathrm{R}_{\text {hid }}(\mathrm{nm}) \times 10^{-3}$ & 4,12 & 4,28 & 3,58 & 3,31 \\
$\mathrm{dQ}_{\mathrm{ef}}\left(\mathrm{Q} / \mathrm{nm}^{3}\right)$ & 6,8 & 6,1 & 5,2 & 6,6 \\
\hline
\end{tabular}


La relación entre el tratamiento con polímeros y las propiedades del suelo es ampliamente afectada por la adsorción de las moléculas de polímero sobre las partículas del suelo. La absorción de polímeros no iónicos (PVP), de cadena flexible, sobre las partículas de arcilla incrementa con el peso molecular y generalmente lleva a la desorción de numerosas moléculas de agua. La adsorción de tales polímeros es usualmente un proceso controlado entropicamente y de tipo irreversible (Wallace y Terry 1998).

Por otro lado, los polímeros negativamente cargados (PAA y PSSNa) tienden a repelerse de la superficie de las arcillas como resultado de las interacciones electrostáticas. Los polímeros aniónicos no tienden a entrar en el espacio interlaminar de los minerales expandidos o exfoliados. Sin embargo, su adsorción es promovida por la presencia de cationes polivalentes que actuan como puentes entre los grupos aniónicos del polímero y los sitios cargados negativamente de las arcillas (Wallace 1998). Además, el incremento de la fuerza iónica de la solución reduce las repulsiones electrostáticas entre el polianión y la superficie de las arcillas (el apantallamiento de las cargas resulta más efectivo en el PAA que en el PSSNa debido a la naturaleza débil de los grupos $-\mathrm{COOH}$ que forman equilibrio en solución) (Rivas et al. 2011).

La alta fuerza iónica del medio tendrá un efecto más marcado sobre el PAA y PSSNa, ya que al verse afectada su densidad de carga se genera una disminución del tamaño del polímero en solución, en consecuencia, al disminuir las interacciones entre los grupos negativamente cargados su radio hidrodinámico disminuye (Szilagyi et al. 2014). Así mismo, la capacidad de retención de iones de los tres polímeros estudiados se ve fuertemente afectada por la presencia de otros iones y por la alta fuerza iónica del medio (Rivas et al. 2011). El efecto de la disminución de la retención promueve la adsorción de los polímeros menos fuertemente cargado, lo que sigue el siguiente orden PVP > PAA > PSSNa (Szilagyi et al. 2014). Sin embargo, estudios de desorción y lavado de los polímeros a horizontes inferiores deberían ser realizados. De igual forma, el efecto sobre la CIC puede verse influenciada fuertemente.

\section{CONCLUSIONES}

A partir de los resultados obtenidos se concluye que los polímeros solubles en agua evaluados (PAA, PSSNA y PVP) son capaces de alterar la composición química de la solución del suelo a concentraciones. relativamente bajas $\left(<10 \mathrm{mgL}^{-1}\right.$ por cada $100 \mathrm{~g}$ de suelo). La tendencia del cambio generado sobre los contenidos de bases intercambiables dependerá de la naturaleza del catión y de la capacidad de formar complejos con los grupos funcionales de la cadena polimérica. Para los iones divalentes, el efecto observado del PSSNa difiere del PAA y PVP para el $\mathrm{Ca}^{2+}$ y $\mathrm{Mg}^{2+}$. En relación a cationes monovalentes, el sodio presentó una interacción débil con los polímeros estudiados. 


\section{AGRADECIMIENTOS}

Los autores agradecen a la Universidad del Valle y a la Universidad de Córdoba por los fondos recibidos.

\section{REFERENCIAS}

\section{Bai, M., Wilske, B., Buegger, F., Esperschuttz,} J., Bach, M., Frede, H.G., and Breuer, L. 2015. Relevance of nonfunctional linear polyacrylic acid for the biodegradation of superabsorbent polymer in soils. Environmental Science Pollution Resourse International 22(7):5444-5452.

Burton, E., Bush, R.T., Sullivan, L., Johnston, S., and Hocking, R. 2008. Mobility of arsenic and selected metals during reflooding of iron- and organic-rich acidsulfate soil, Chemical Geology, 253:6473 .

Callebaut, F., Gabriels, D., and De Boodt, $M$. 1979. The effect of polymer structure on soil physico-chemical properties and soil water evaporation, Journal of Chemistry, Technology and Biotechnology 29:723729.

Combatt, E., Martínez, Z., Cabrales, E., Martínez, G., Castillo, C. and Palencia, M. 2004. Caracterización fisicoquímica y mineralógica de los suelos sulfatados ácidos en el transecto San Carlos-CotorraCarrillo. Universidad de Córdoba.

Combatt, E., Martinez, Z., and Palencia, M. 2008. Generación de acidez por oxidación de pirita en suelos sulfatados ácidos interiores de clima cálido, Temas agrarios, 13:32-39.

Dorraji, S.S., Golchin, A., and Ahmadi, S. 2010. The effects of hydrophilic polymer and soil salinity on corn growth in sandy and loamy soils clean, Soil, Air, Water, 38: $584-591$.

Guiwei, Q., De Varennes, A., and CunhaQueda, C. 2008. Remediation of a mine soil with insoluble polyacrylate polymers enhances soil quality and plant growth. Soil Use and Management, 24:350-356.

Instituto Geográfico Agustin Codazzi (IGAC) 2006. Manual de análisisquímico de suelos, IGAC, Bogotá.

LI, X., HE, J.Z., Hughes, J.M., LIU, Y.R., and Zheng, Y.M. 2014. Effects of superabsorbent polymers on a soil-wheat (Triticumaestivum L.) system in the field, Applied Soil Ecology 73:58-63.

Mohammed, M.A., Eldeen Mohammed, R.D., and Fathy, A. 2013. Conditioning Effect of Different Absorbant Polymers on Physical and Chemical Properties of Sandy Soil, Journal of Functional and Environmental Botany, 3:82-93.

\section{Mukhopadhyay, R., Gajrib, PR., and Chaudhary} ,MR. 1994. Synthesis of a soil conditioner from acrylic waste and its effect on aggregate stability and moisture retention in two soils. Arid Soil Research and Rehabilitation 8(2):179-186. 
Nightingale, E.R. 1959. Phenomenological Theory of Ion Solvation.Effective Radii of Hydrated lons. The Journal of Physical Chemistry, 63:1381-1387.

Pardo, S., Suárez, H., y Pertuz, V. 2009. Interacción de los suelos sulfatados ácidos con el agua y sus efectos en la sobrevivencia del bocachico (Prochilodusmagdalenae) en cultivo, Revista Colombiana de Ciencias Pecuarias, 22:619-631.

Powell, B., Martens, M. 2005. A review of acid sulfate soil impacts, actions and policies that impact on water quality in Great Barrier Reef catchments, including a case study on remediation at East Trinity Marine, Pollution Bulletin, 51:149-164.
Rivas, B., Pereira, E., Palencia, M., y Sanchez, J. 2011. Water-soluble functional polymers in conjunction with membranes to remove pollutant ions from aqueous solutions, Progress in Polymer Science, 36:294-322.

\section{Szilagyi, I., Trefalt, G., Tiraferri, A., Maroni, P.,} and Borkovec, M. 2014. Polyelectrolyte adsorption, interparticle forces, and colloidal aggregation. Soft Matter 15(10):2479-2502.

Wallace, A., Wallace, G. 1986. Effects of very low rates of synthetic soil conditioners on soils, Soil Science, 141:324-327.

Wallace, A, Terry, R. 1998. Handbook of Soil Conditioners: Substances That Enhance the Physical Properties of Soil: Substances That Enhance the Physical Properties of Soil. CRC Press. 600 p. 